\title{
Performance Evaluation of a High-Frame Rate 3D Range Sensor for Construction Applications
}

\author{
Alan M. Lytle, Itai Katz, and Kamel S. Saidi
}

\begin{abstract}
The NIST Construction Metrology and Automation Group (CMAG) has ongoing research in the application of laser imaging technologies in construction. Previous work has primarily focused on terrestrial LADAR scanning for applications such as terrain characterization, earthmoving analysis, and targeted object localization. The LADAR systems used in those research efforts can be characterized by long scan intervals and dense point cloud returns. Current research includes the investigation of a new class of commerciallyavailable, optical range image sensors for construction mobility applications. These flash LADAR systems (or 3D range cameras) yield a low resolution $3 \mathrm{D}$ range map and intensity image at up to 30 frames per second, and show promise for applications such as obstacle avoidance and object detection and tracking. This paper summarizes recent CMAG research with these sensors including calibration and performance evaluation. Future work involving crane obstacle avoidance and positioning is also discussed.
\end{abstract}

Index Terms - construction automation, LADAR imaging, 3D range camera, performance evaluation

\section{INTRODUCTION}

\section{A. LADAR Performance Evaluation}

The NIST Construction Metrology and Automation Group (CMAG) has been researching the use of terrestrial or 'tripod mounted' LADAR (Laser Detection and Ranging) in construction since 1999. Initial work led to the conclusion that the following were needed to characterize LADAR systems and develop confidence limits for the LADAR data and associated derivative information:

- clarification of commonly used terminology,

- development of standard test protocols and reporting procedures,

- facilitation of "factory floor" calibrations through the use of traceable artifacts and standard procedures, and the

- availability to manufacturers and end users of a neutral facility for testing/calibration under environmentally controlled conditions.

The need for LADAR standards and references to related

A. M. Lytle, and K. S. Saidi are with the National Institute of Standards and Technology, Gaithersburg, MD 20899, USA (corresponding author: A. M. Lytle, tel: 301-975-6048; fax: 301-975-6122; e-mail: alan.lytle@nist.gov).

I. Katz is a graduate student at Stanford University, Stanford, CA 94305, USA (e-mail: itai@stanford.edu). terrestrial LADAR performance evaluation work is provided in [1]. Prior work for evaluating flash LADAR systems is provided in [2] and [3]. To date, NIST has hosted two workshops for manufacturers and end-users of LADAR systems related to the development of LADAR standards. The proceedings of the 2003 and 2005 workshops are available as [4] and [5], respectively. The next workshop will be held at the NIST Gaithersburg, MD campus in early 2006.

\section{B. Flash LADAR for Construction}

The FIATECH Intelligent and Automated Construction Job Site Tactical Plan ${ }^{1}$ envisions that

Construction sites will become more 'intelligent and integrated' as materials, components, tools, equipment, and people become elements of a fully sensed and monitored environment. Location and status of all materials, equipment, personnel, and other resources will be continuously tracked on site, thus enabling a 'pull' environment where needed resources are delivered on demand. Automation of construction processes will augment manual labor for hazardous and labor-intensive tasks such as welding and high-steel work. Construction job sites will be wirelessly networked with sensors and communications technologies that enable technology and knowledge-enabled construction workers to perform their jobs quickly and correctly. [6]

In order to realize robust field-automation on dynamic and cluttered construction sites, advanced sensing capabilities are required to provide machine situational awareness.

Drawing on research experience in construction site monitoring and autonomous mobility, Stone et al [7] describe the "ultimate construction site sensor" as a LADAR system having the following characteristics:

$$
\begin{array}{ll}
\text { Illumination Source: } & \text { eyesafe } \\
\text { Field of View (FOV): } & 60^{\circ} \times 60^{\circ} \\
\text { Range Uncertainty: } & \pm 1 \mathrm{~mm} \text { for }<15 \mathrm{~m} \\
& \pm 3 \mathrm{~mm} \text { for }<100 \mathrm{~m} \\
& <0.03^{\circ} \\
\text { Angular Resolution: } & >10 \mathrm{~Hz} \\
\text { Frame Rate: } & >19
\end{array}
$$

\footnotetext{
${ }^{1}$ The two NIST authors on this paper are members of the FIATECH Intelligent and Automated Construction Job Site working team.
} 


$$
\begin{array}{ll}
\text { Size: } & \sim \text { Coffee Cup } \\
\text { Cost: } & <\$ 1000 \text { USD }
\end{array}
$$

While no LADAR instrument currently meets these combined specifications, new systems are commercially available which enable high frame rate data capture, albeit at higher range uncertainty and lower angular resolution than desired. Unlike the more common terrestrial scanning LADARs currently found on construction sites, these focal plane array (FPA), or 'flash' LADAR systems do not require a scanning mechanism. Instead flash LADARs use a broad field illumination source and an FPA detector, such that the entire range image is completely acquired simultaneously in one burst. The source is commonly a laser, but for short range applications $(<10 \mathrm{~m})$ a bank of light emitting diodes (LEDs) can be used.

Most research and development programs for flash LADAR systems are devoted to military demand for real-time, medium accuracy sensors. Several infrared (IR) LED-based systems are now commercially available in either engineering prototype or first generation production models. These systems show promise for automation applications, but they are limited by higher range uncertainty, susceptibility to ambient lighting conditions, and lower spatial information content in the range image due to current FPA size limitations. Initial research in the use of these instruments for mobile robot navigation applications is provided in [8] and [9].

Despite the above limitations, the groundwork established from an examination of these sensors will provide an important baseline for continued research in LADAR-related construction automation efforts, particularly as defense-related development efforts continue to drive down the cost of everimproving flash LADAR systems for dynamic imaging applications.

\section{Paper Outline}

This paper presents initial CMAG efforts to characterize short range flash LADAR systems. Section II defines terminology related to performance characteristics, describes the flash LADAR used in the testing, and discusses the test equipment and methods used to perform an initial system calibration and to determine performance characteristics. Section III describes the tests developed for the specific characteristics - range uncertainty and instrument resolution (horizontal) - and sections IV and V provide conclusions and a description of future work, respectively. It is important to note that this paper does not provide definitive results of tests of any particular device. Rather, the motivation is to help foster the development of standard tests and evaluation criteria for the flash LADAR user community.

\section{EXPERIMENTAL PARAMETERS AND PROCEDURES}

\section{A. Definition of Terms}

The need for commonly defined terms for describing LADAR performance characteristics was evident at the first two NIST LADAR workshops. While many terms such as accuracy, uncertainty, and repeatability already have universally accepted definitions that were developed by standards organization (e.g., [10]) the application of those terms to describe particular LADAR attributes remains open. In addition, the nature of the LADAR measurements and end products necessitates new terminology not currently standard in the literature. One of the main goals of the 3rd NIST LADAR workshop will be to finalize a draft set of terminology for the user community. As it pertains to the efforts described in this paper, the following definitions are proposed:

Range Uncertainty: The uncertainty associated with a distance measurement reported by an instrument to a given object of interest (or target). Uncertainty, as defined in [10], is a parameter associated with the result of a measurement that characterizes the dispersion of the values that could reasonably be attributed to the measurand. Range uncertainty as a function of target range, target reflectance, and target angle of incidence is of particular interest.

Instrument Resolution: The smallest discernable separation between two objects. Instrument resolution can be divided into three components: range, horizontal, and vertical.

\section{B. Experimental Setup}

A custom test apparatus was designed and assembled for evaluating the flash LADAR. The apparatus consists of an 8 $\mathrm{m}$ long aluminum linear rail supported by three stands and three pairs of lockable casters (see Fig. 1). Mounted on top of the rail are three manually operated bearings that can be secured in place anywhere along the length of the rail. Attached to each bearing is a mechanism which provides two degrees of freedom (pitch and yaw) to the load (see Fig. 2). One bearing holds the flash LADAR, while the other two bearings hold targets that are to be imaged. The flash LADAR is mounted onto a manually-operated $\mathrm{XY}$ linear stage combined with a rotary stage.

The targets mounted onto the linear bearings are $21.6 \mathrm{~cm}$ ( 8.5 in) by $30.5 \mathrm{~cm}$ (12 in) aluminum plates. A gray card (with $18 \%$ reflectance) is attached to each of the plates.

The test apparatus was designed to allow us to achieve the following motions (refer to Fig. 3):

a. Move 2 targets apart within the same plane to determine horizontal resolution.

b. Move 2 targets apart along the normal vectors to their surfaces to determine range resolution.

c. Rotate a target along its longitudinal axis to determine effects of angle of incidence.

d. Move the flash LADAR and/or targets in a straight line away from and towards each other to determine effect of distance.

e. Rotate the flash LADAR along its optical center (not shown in figure) to test specified parameters within the LADAR FOV. 


\section{Instrument Description}

The flash LADAR used for these experiments is a commercially-available, phase-based AM-homodyne device which uses a bank of IR LEDs (870 nm wavelength) for the illumination source. The source is modulated at $20 \mathrm{MHz}$ yielding an unambiguous range of $7.5 \mathrm{~m}$. The FPA is 160 pixels $x 124$ pixels with a corresponding nominal field of view (FOV) of $43^{\circ} \times 46^{\circ}$. Data returned from the device includes range, intensity, and amplitude per pixel at a frame rate of approximately $30 \mathrm{~Hz}$. Settings for the integration time, amplitude threshold, and distance offset are user-controlled.

\section{Initial Calibration}

Prior to experimental use, the flash LADAR is calibrated to determine the intrinsic parameters of the lens and CCD and the measured distance offset correction. The intrinsic parameters are useful for eliminating image distortion, verifying horizontal and vertical FOV, and connecting an arbitrary pixel to its corresponding point in space given an origin and set of bases. The distance offset correction minimizes instrument range bias, and is determined along the center axis of the device (i.e. normal to the image plane). Any calculations that assume a planar target must compensate for the fact that geometric distance to a target varies with angle of incidence. Since our sampling mask was localized at the center and small relative to the pixel size of the FPA, this effect was ignored.

\section{1) Intrinsic Parameters}

The Zhang algorithm [11], as implemented in the Intel OpenCV library [12], was used to characterize the instrument's intrinsic parameters. Fifteen intensity images of a checkerboard calibration target where obtained at various orientations and positions in the FOV. For each image, a $6 \mathrm{x}$ 5 grid of corner points was selected.

The calibration algorithm returns the camera matrix,

$$
\left[\begin{array}{ccc}
f / S_{x} & 0 & \text { center }_{x} \\
0 & f / S_{y} & \text { center }_{y} \\
0 & 0 & 0
\end{array}\right]
$$

where $f / S_{x}$ and $f / S_{y}$ are the focal length in units of horizontal and vertical pixels, and center $\mathrm{x}_{\mathrm{x}}$ and center $\mathrm{y}_{\mathrm{y}}$ are the $\mathrm{x}$ and $\mathrm{y}$ pixel array components of the principal point, respectively. The algorithm also returns the distortion vector,

$$
\left[\begin{array}{llll}
k_{1} & k_{1} & d_{1} & d_{2}
\end{array}\right]
$$

where $\mathrm{k}_{1}$ and $\mathrm{k}_{2}$ are radial distortions due to the error in the lens and $\mathrm{d}_{1}$ and $\mathrm{d}_{2}$ are tangential errors associated with imperfect centering of the lens with respect to the CCD.

From these data, an expression for the horizontal FOV and the vertical FOV is given by equations (1) and (2), respectively as follows:

$$
\begin{aligned}
& F O V_{h}=\tan ^{-1}\left[\frac{\text { center }}{\left(f / S_{x}\right.}\right]+\tan ^{-1}\left[\frac{\text { width }- \text { center }}{\left(f / S_{x}\right)}\right] \\
& F O V_{v}=\tan ^{-1}\left[\frac{\text { center }}{\left(f / S_{y}\right)}\right]+\tan ^{-1}\left[\frac{\text { height }- \text { center }}{\left(f / S_{y}\right)}\right]
\end{aligned}
$$

The calibration algorithm was repeated for every combination of 14 images from the available 15 images. The mean and standard deviation for each parameter was derived from these 14 data sets (see Table I). From the values in Table I the FOV is calculated using equations (1) and (2) and the results are shown in Table II.

The theoretical FOV was determined by evaluating (1) and (2) with the manufacturer's specified parameters. Since a principal point was not specified, a location in the middle of the CCD was assumed. This assumption accounts for the discrepancy between the theoretical and observed values.

\section{2) Distance Offset Correction}

The next step in the LADAR calibration was to set the correction factor for the range bias. This is a two step process because the measured range is a function of the integration time of the LADAR. Fig. 4 illustrates the effects of varying the integration time for a target placed at $4 \mathrm{~m}$ from the LADAR.

Fig. 5 shows the effect of varying integration time for ranges of $1 \mathrm{~m}$ through $7 \mathrm{~m}$ (the range error was not bias corrected). Fig. 6 is an expanded view of the region of interest where the divergence of the error curves was minimized. From this graph an integration time of $20.5 \mathrm{~ms}$ was selected. Range bias was then experimentally obtained at $4 \mathrm{~m}$ and a distance offset correction was calculated.

\section{E. Performance Tests}

Two performance tests were conducted using the test apparatus. Each test was performed at ranges of $1 \mathrm{~m}$ through $7 \mathrm{~m}$ at intervals of $1 \mathrm{~m}$ with the targets at a zero angle of incidence and using the $18 \%$ reflectance card. All tests were conducted indoors under ambient lighting (fluorescent) conditions. Ranges to the targets were measured using a laser distance meter with an uncertainty of $\pm 1.5 \mathrm{~mm}$ over a range of $0.2 \mathrm{~m}$ to $200 \mathrm{~m}$.

\section{1) Range Uncertainty}

Two targets were mounted side by side to provide a 40.5 $\mathrm{cm}$ (16 in) x $25.4 \mathrm{~cm}$ (10 in) planar surface. At each range $\mathrm{N}=1000$ images were obtained and range data were sampled from a $7 \times 3$ pixel mask at the center of the targets. The sampling was repeated two additional times at each range for a total of three sets per range increment.

\section{2) Instrument Resolution (Horizontal)}

Two targets were mounted side by side on slide bearings to provide a $40.5 \mathrm{~cm}$ (16 in) x $25.4 \mathrm{~cm}$ (10 in) planar surface and to enable separation normal to the LADAR. A third target was mounted behind the first two at a distance of 3 times the 
standard deviation of the measured range obtained during the range uncertainty test. The plates were then incrementally separated starting with a gap of 0.5 times the calculated pixelwidth for the given range in steps of 0.25 times the calculated pixel-width. At each increment, $\mathrm{N}=100$ images were obtained and range data were sampled from a $4 \times 2$ pixel mask centered on the gap. The mean of this trimmed range image was then processed by a Laplacian of Gaussian (LoG) edge detector. If two distinct lines were detected, the two separated targets were declared to be detected. This process was repeated ten times for each gap increment.

\section{RESULTS}

\section{A. Range Uncertainty}

The results of the range error measurement are shown in Fig. 7. Range uncertainty increased with range and the range at which the distance offset correction was set was the most accurate (but not the most precise). The mean error appeared to oscillate as a function of range but more testing is required to determine whether there is a repeatable pattern.

Using a single integration time over the range of testing is not optimal but was done to limit the variables in these first tests. Since there is interest in using these devices for mobile robot navigation in unstructured environments, it is expected that integration time will have to be adjusted in real-time to account for varying ambient conditions and the returned signal strength. The effect of dynamically changing those parameters will have to be known. Further work in analyzing the range error as a function of the range selected for measuring the distance offset correction is also warranted.

\section{B. Instrument Resolution (Horizontal)}

Establishing a repeatable method of measuring the instrument resolution was problematic. Initial efforts examining the change in measured range standard deviation over the pixel mask worked well at short ranges $(<3 \mathrm{~m})$ but proved difficult and not repeatable at longer ranges when faced with the higher levels of range uncertainty. This might have been overcome by significantly increasing the sample size, but without automating the data collection and target repositioning the standard deviation approach was impractical.

The edge detection approach was fast and clear pass/fail rules were easily established. Since the pixel footprint is known to expand with range, using increments of $25 \%$ of the nominal pixel-widths at each range was useful in minimizing the manual repositioning of the targets. The results of the horizontal resolution tests are shown in Table III. Future work on exploring alternative gap detection methods for instrument resolution testing is recommended.

\section{CONCLUSIONS}

The test apparatus functioned as designed. Recommended changes include mounting the LADAR on a computer controlled pan and tilt system for FOV testing, and changing the target holder to including a linear stage to facilitate separating targets during the resolution testing. A computercontrolled target placement system which would enable automatic control of target angle and separation would be useful.

\section{FUTURE WORK}

Future work will include revising and expanding the test methods described in this paper to evaluate the following:

- Range as a function of target angle of incidence and target reflectance,

- Instrument resolution (range, vertical, and horizontal)

- Range uncertainty and instrument resolution as a function of a target's position within the instrument's field of view,

- Angular uncertainty,

- Range, instrument resolution, and angular uncertainty as a function of ambient conditions.

Once the evaluation has been expanded to include the above parameters, the test methods will also be applied to multiple flash LADAR units from the same and different manufacturers.

Finally, the results of the above experiments will be applied to ongoing CMAG efforts aimed at using flash LADAR as an obstacle avoidance sensor for a robotic crane, and as a guidance sensor for autonomous structural steel pick-andplace operations using the same crane (see Fig. 8). An outcome of this effort will also be applied to using flash LADAR for obstacle detection on existing construction equipment.

\section{REFERENCES}

[1] Cheok, G. S., Stone, W. C., and Lytle, A., "Standards Requirements for LADARs?", in Laser Radar Technology and Applications X, edited by G. W. Kamerman, Proceedings of SPIE Vol. 5791, pp. 250-261, 2005.

[2] Brian Miles, Jay Land, Andrew Hoffman, William Humbert, Brian Smith, Andrew Howard, Joe Cox, Mike Foster, Dave Onuffer, Sammie Thompson, Tom Ramrath, Clarke Harris, and Paul Freedman, "Field Testing Protocols for Evaluation of 3D Imaging Focal Plane Array Ladar Systems," in Laser Radar Technology and Applications VII, edited by G. W. Kamerman, Proceedings of SPIE Vol. 4723, pp. 43-56, 2002.

[3] T. C. Monson, J. W. Grantham, and S. W. Childress, J. T. Sackos, R. 0. Nellums, and S. M. Lebien, "Characterization of Scannerless LADAR," in Laser Radar Technology and Applications IV, edited by G. W. Kamerman, Proceedings of SPIE Vol. 3707, pp. 409-420, April 1999.

[4] G. S. Cheok, Ed. "Proceedings of the LADAR Calibration Facility Workshop, June 12-13, 2003," NISTIR 7054 (CD-ROM), National Institute of Standards and Technology, Gaithersburg, MD, October, 2003.

[5] G. S. Cheok, Ed. "Proceedings of the LADAR Calibration Facility Workshop, March 15-16, 2005," (NISTIR to be published), National Institute of Standards and Technology, Gaithersburg, MD, October, 2005.

[6] FIATECH (2004). "Capital Projects Technology Roadmap: Element 4 Tactical Plan, Intelligent and Automated Construction Job Site (IACJS)" www.fiatech.org.

[7] W. Stone, M, Juberts, N. Dagalakis, J. Stone, J. Gorman. "Performance Analysis of Next-Generation LADAR for Manufacturing, Construction, and Mobility," NISTIR 7117, National Institute of Standards and Technology, Gaithersburg, MD May 2004.

[8] Weingarten, J., Gruener, G., and Siegwart, R.. "A State-of-the-Art 3D Sensor for Robotic Navigation." Proceedings IROS Vol 3, pp. 21552160, 28 Sept.-2 Oct. 2004 
[9] Roger Bostelman, Tsai Hong, and Raj Madhavan, "Experiments Toward Non-contact Safety Standards for Automated Industrial Vehicles," in Unmanned Ground Vehicle Technology VII, edited by Grant R. Gerhart, Charles M. Shoemaker, Douglas W. Gage, Proceedings of SPIE Vol. 5804, pp. 67-77, 2005.

[10] International Vocabulary of Basic and General Terms in Metrology, International Organization for Standardization, 1993.

[11] Z. Zhang, "A flexible new technique for camera calibration”, IEEE Transactions on Pattern Analysis and Machine Intelligence, Vol. 22, Issue 11, pp. 330-334, 2000.

[12] Intel OpenCV Computer Vision Library $(\mathrm{C}++)$, http://www.intel.com/research/mrl/research/opencv/.

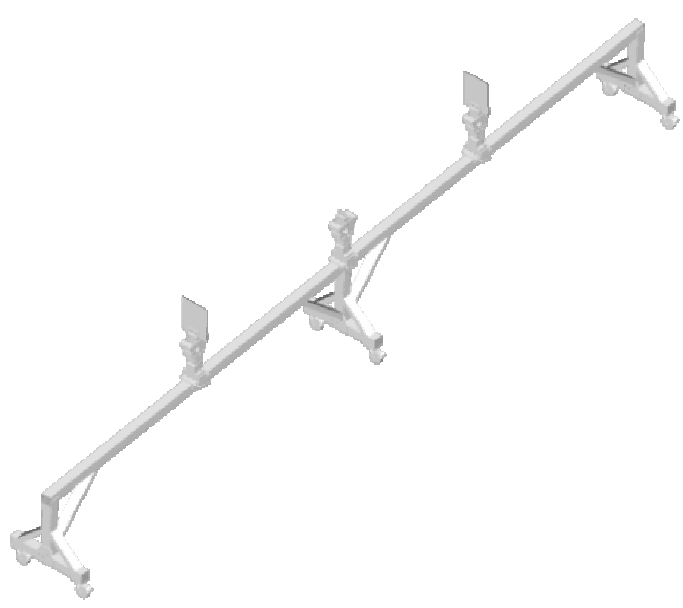

Fig. 1. Test Apparatus. This is a rendering of the $8 \mathrm{~m}$ long test apparatus shown with the three linear bearings attached with two targets at either end of the rail and the flash LADAR mounted in the center.

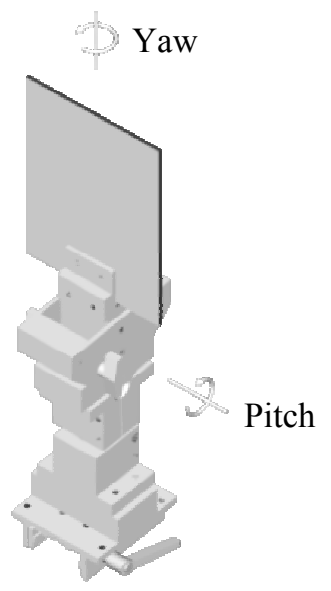

Fig. 2. Target Mount. This is a rendering of one of the two target mounts that can be attached to the rail of the test apparatus.
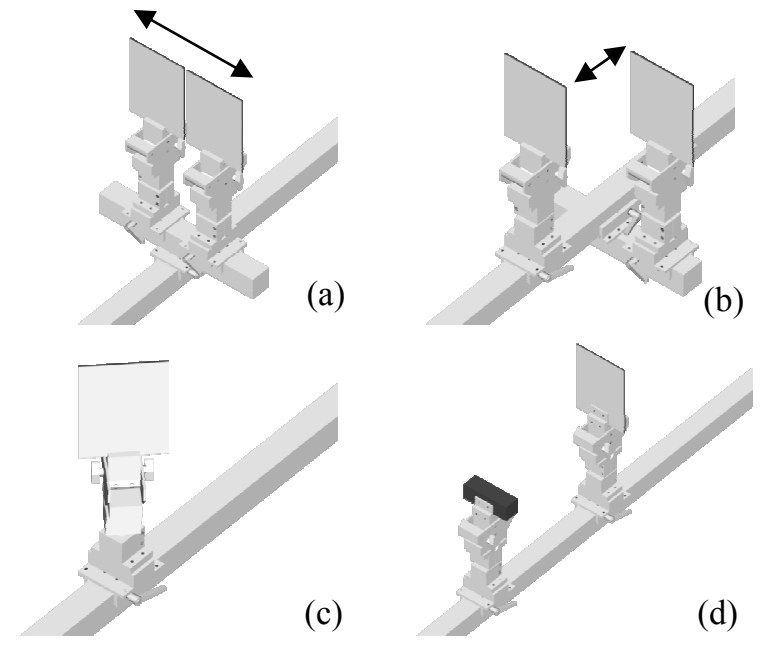

Fig. 3. Target configurations on the test apparatus.

TABLE I

CAMERA CALIBRATION PARAMETERS

\begin{tabular}{ccc}
\hline \hline Parameter & mean & std \\
\hline $\mathrm{f} / \mathrm{S}_{\mathrm{x}}$ & 211.2 & 0.97 \\
$\mathrm{f} / \mathrm{S}_{\mathrm{y}}$ & 151.2 & 0.64 \\
center $_{\mathrm{x}}$ & 75.3 & 0.51 \\
center $_{\mathrm{y}}$ & 64.7 & 0.47 \\
$\mathrm{k}_{1}$ & -0.1696 & 0.0071 \\
$\mathrm{k}_{2}$ & 0.2490 & 0.0285 \\
$\mathrm{~d}_{1}$ & 0.0089 & 0.0007 \\
$\mathrm{~d}_{2}$ & -0.0027 & 0.0006 \\
\hline \hline
\end{tabular}

TABLE II

CAMERA FOV

\begin{tabular}{lccc}
\hline \hline FOV & $\begin{array}{c}\text { Theoretical } \\
(\mathrm{deg})\end{array}$ & $\begin{array}{c}\text { Observed } \\
(\mathrm{deg})\end{array}$ & std \\
\hline horizontal & 42.8 & 41.5 & 0.2 \\
vertical & 46.1 & 44.6 & 0.2 \\
\hline \hline
\end{tabular}




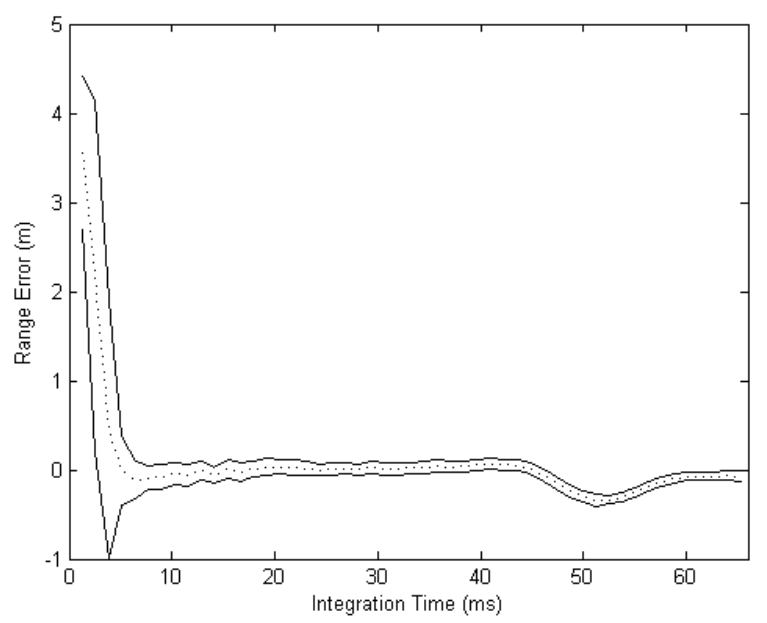

Fig. 4. Range error (with offset removed) vs. integration time for a target at 4 $\mathrm{m}$. Range error based on $\mathrm{N}=100$ image samples and $7 \times 3$ pixels sampled per range image. Solid lines indicate uncertainty $( \pm 1$ ó).

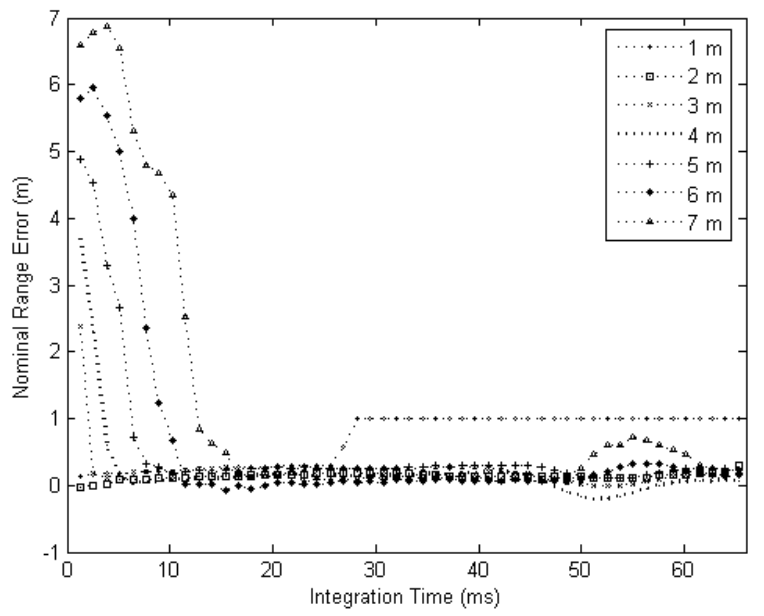

Fig. 5. Nominal (no bias correction) range error vs. integration time. Flat line at $1 \mathrm{~m}$ indicates saturation limit. Nominal range error based on $\mathrm{N}=100$ image samples and $7 \times 3$ pixels sampled per range image.

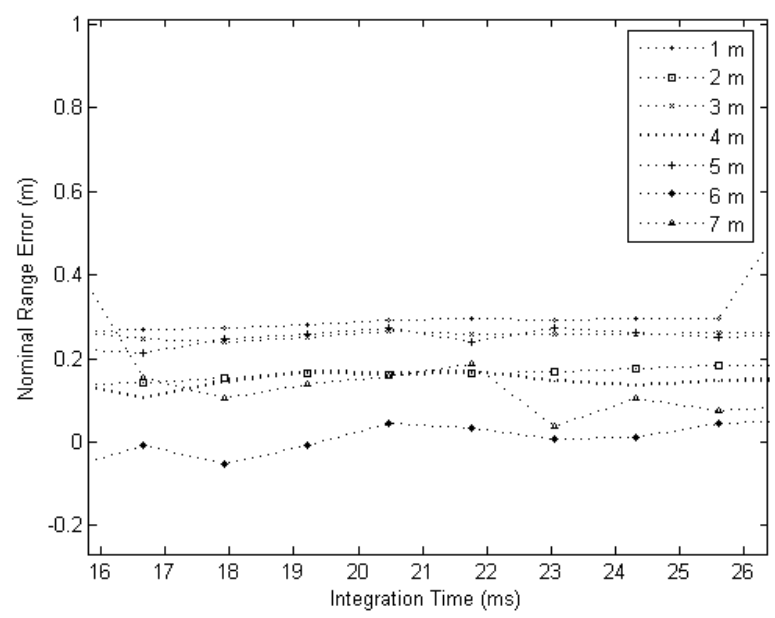

Fig. 6. Nominal (no bias correction) range error vs. integration time. Region of interest, in Fig. 5, expanded.

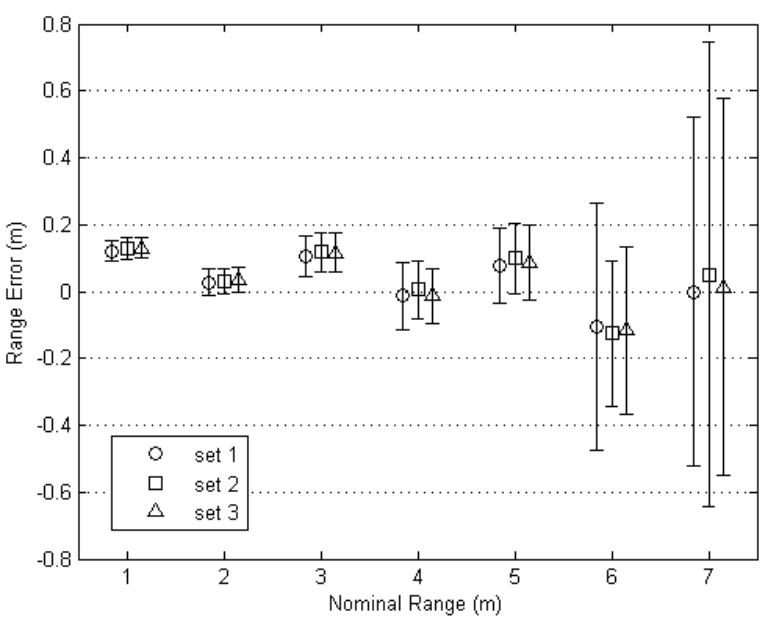

Fig. 7. Range Uncertainty test results for $\mathrm{N}=1000$ image samples and $7 \times 3$ pixels sampled per range image. All samples are with the target angle of incidence $=0^{\circ}$ and target reflectance $=18 \%$. Three sets for each range increment. Plotted points are offset slightly for visibility. Error bars indicate uncertainty $( \pm 1 \sigma)$.

TABLE III

RESULTS OF THE HORIZONTAL RESOLUTION TEST

\begin{tabular}{ccc}
\hline \hline Range (m) & $\begin{array}{c}\text { Gap Size Detected } \\
\text { Minimum 70 \% } \\
\text { Success Rate } \\
\text { (Pixel-width) }\end{array}$ & $\begin{array}{c}\text { Measured } \\
\text { Gap Size } \\
(\mathrm{mm})\end{array}$ \\
\hline 1 & 1.5 & 7 \\
2 & 0.5 & 5 \\
3 & 2.0 & 27 \\
4 & 1.25 & 23 \\
5 & 2.0 & 45 \\
6 & 1.75 & 47 \\
7 & 1.25 & 40 \\
\hline \hline
\end{tabular}

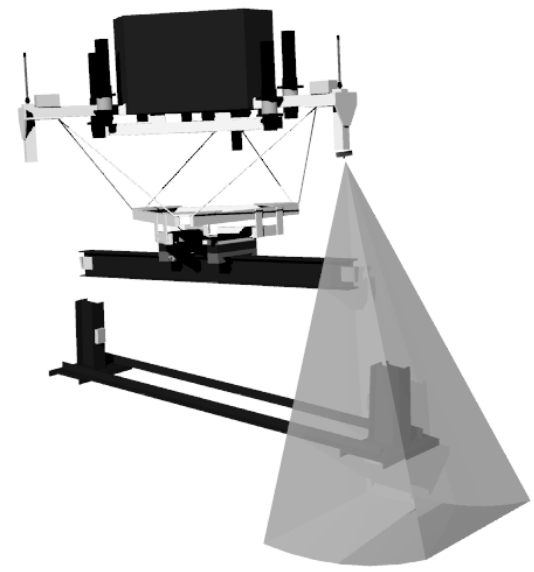

Fig. 8. Concept rendering of NIST RoboCrane using flash LADAR for docking guidance and obstacle avoidance. 